\title{
Análise comparativa de deposições de filmes de óxido de grafeno por spray ultrassônico e convencional
}

\section{Comparative analysis of depositions of graphene oxide films by ultrasonic and conventional spray}

\author{
Andreza Menezes Limaํㅜ, Thebano Emílio de Almeida Santos ${ }^{2}$, \\ Rodrigo Amaral de Medeiro ', Leila Rosa de Oliveira Cruz ${ }^{1}$, \\ Wagner Anacleto Pinheiro ${ }^{1}$
}

\begin{abstract}
${ }^{1}$ Instituto Militar de Engenharia - IME - Praça General Tibúrcio, 80, Urca, CEP: 22290-270, Rio de Janeiro, RJ, Brasil. ${ }^{2}$ Centro de Tecnologia da Informação Renato Archer - CTI Renato Archer, Rodovia Dom Pedro I, Km 143,6, CEP: 13069-901, Campinas, SP, Brasil. e-mail: andrezamenezeslima@gmail.com, thebano.santos@cti.gov.br, digfisic@gmail.com, leilacruz@ime.eb.br, anacleto@ime.eb.br.
\end{abstract}

\begin{abstract}
RESUMO
A técnica de deposição de filmes por spray destaca-se por sua versatilidade, simplicidade e eficiência. Neste trabalho, dois equipamentos de spray distintos foram empregados para produção de filmes de óxido de grafeno: o primeiro, um equipamento industrial para produção por método ultrassônico e o segundo, uma adaptação realizada com um aerógrafo, um solenoide, um circuito elétrico e um programa em linguagem $\mathrm{C}++$, denominado de método convencional. Os filmes obtidos foram caracterizados com respeito à espessura, resistividade elétrica, transmitância e morfologia. As amostras produzidas pelo método convencional apresentaram menores espessuras e maiores transmitâncias, sendo, desse modo, mais adequadas para aplicações optoeletrônicas. Já as menores resistências de folha foram obtidas pelo método de spray ultrassônico. A morfologia dos filmes aponta melhor uniformidade para as amostras obtidas por meio da técnica ultrassônica. A análise comparativa dos resultados indicou que o equipamento convencional, apesar de ser muito mais simples e de baixo custo, foi capaz de alcançar resultados tão satisfatórios quanto os obtidos em um equipamento industrial de spray ultrassônico. A grande vantagem do equipamento industrial está na possibilidade de produção de várias amostras simultaneamente, enquanto o convencional adaptado tem como benefício a rapidez, possibilidade de deposição com folhas de óxido de grafeno maiores e acessibilidade da produção e operação do sistema.
\end{abstract}

Palavras-chave: óxido de grafeno; deposição por spray; filmes finos.

\begin{abstract}
The spray technique for thin film deposition stands out for its versatility, simplicity and efficiency. In this work, two different spray techniques, conventional and ultrasonic, were used for the production of graphene oxide films, the first one was an industrial equipment for ultrasonic spray production and the second, an adaptation performed with an airbrush, a solenoid, a circuit electric and a program in $\mathrm{C}++$ language, called conventional spray. The films obtained were characterized with respect to thickness, electrical resistivity, transmittance and morphology. The samples produced by the conventional method presented thicknesses and transmittances more suitable for electronic applications, since the smaller sheet resistances were obtained by the ultrasonic spray method. The morphology of the films shows a better uniformity for the samples obtained by means of the ultrasonic spray technique. The comparative analysis of the results indicated that the conventional spray equipment, although much simpler and low cost, was able to achieve results as satisfactory as those obtained in an industrial ultrasonic spray equipment. The great advantage of the industrial equipment is the possibility of producing several samples simultaneously, while the conventional spray adapted has the benefit of speed, deposition possibility with larger graphene oxide sheets and accessibility of the production and operation of the system.
\end{abstract}

Keywords: graphene oxide; transparent and conductive films; methods of deposition. 


\section{INTRODUÇÃO}

Variadas metodologias são aplicadas para a produção de filmes e revestimentos de óxido de grafeno (graphene oxide - GO) e óxido de grafeno reduzido (reduced graphene oxide - rGO). Dentre as diversas técnicas amplamente estudadas, spray coating se destaca por sua versatilidade e compreensibilidade de aplicação.

O spray convencional é um método amplamente aplicado na indústria, facilmente adaptável para produções em larga escala, capaz de recobrir diversos substratos, de variados formatos e com grande variabilidade de parâmetros, permitindo obtenção de filmes com distintas morfologias. O aparato da técnica abrange uma pistola de spray ou um aerógrafo e um gás para transporte da solução. Se comparado a outros métodos de deposição, é extremamente simples e de baixo custo [1]

Porém, a deposição por spray convencional apresenta limitações quanto ao controle de espessura, o que pode ser crítico na obtenção de filmes com resistividade e transparência adequadas. Na técnica de spray ultrassônico uma névoa de baixa velocidade é gerada pela energia vibracional do ultrassom e se condensa sobre o substrato. A frequência do gerador de partículas ultrassônico está inversamente relacionada ao diâmetro das gotículas produzidas, o que aumenta o controle da espessura dos filmes produzidos [2].

$\mathrm{Na}$ atualidade, vários estudos têm envolvido a técnica de spray para formação de filmes nas mais diversas aplicabilidades. PU et al. (2013) [3] produziram filmes compósitos de grafeno e nanotubos de carbono pelo método supracitado em substratos metálicos, para controle da molhabilidade de água. Foi utilizado um aerógrafo com nitrogênio como gás de arraste $(0,2 \mathrm{MPa})$. Após a deposição, os substratos foram aquecidos a $50^{\circ} \mathrm{C}$ para eliminação do solvente. Os filmes obtidos eram contínuos, hidrofóbicos, condutores e com baixa adesão de moléculas de água.

LI et al. (2015) [4] estudaram a aplicação de filmes de grafeno como barreira de umidade em embalagens poliméricas para alimentos. Os autores demonstraram que múltiplas camadas obtidas por spray apresentaram propriedades satisfatórias para tal fim. IBRAHIM et al. (2018) [5], estudaram a produção de filmes de GO por spray coating para separação de gases, demonstrando ser um método eficiente, econômico, escalável e com características muito similares ao método tradicional, que é a filtragem. Nesse caso, o autor utilizou ar comprimido como gás de arraste e o volume de GO depositado para controle da espessura do filme. Já MOON et al. (2013) [6] exploraram a aplicação de spray de grafeno como camada protetora para eletrodos condutores e transparentes flexíveis, comprovando o incremento da hidrofobicidade e da resistência de folha no material, por meio da deposição de 3 camadas de GO. BAYER et al (2017) [7] examinaram a aplicação de GO por spray diretamente na camada eletrocatalítica em células a combustível. Os autores concluíram que a diminuição da espessura da camada, devido ao emprego dessa técnica, melhora significativamente a densidade de potência do dispositivo.

NAGAVOLU et al. (2016) [8] utilizaram filmes de rGO obtidos pela técnica supracitada para aplicação como contraeletrodo em células solares sensibilizadas por corante (DSSC), alcançando eficiência em torno de $4 \%$, valores considerados promissores, dada a facilidade do procedimento de produção. SHI et al. (2015) [9] estudaram filmes de rGO para aplicação como filmes transparentes e condutores, seguindo o revestimento por spray simples, obtendo valores de resistência de folha de $58 \mathrm{k} \Omega / \square$ e transmitância de $68,69 \%$, resultados abaixo do ideal para tal aplicação, mas com grande potencial. TUNG et al. (2018) [10] produziram filmes transparentes e condutores de rGO por spray ultrassônico com resistência de folha de $1,45 \times 10^{7} \Omega / \square$ e transmitância de $87 \%$.

A grande maioria dos estudos reportados tem em comum a utilização de um aerógrafo como instrumento para a realização da técnica de spray. Quando a pistola é acionada manualmente, as chances de problemas com a reprodutibilidade dos filmes obtidos são maiores. Por conseguinte, é importante destacar a utilização de equipamento automatizados, capazes de controlar todas as variáveis. Para aplicações como filmes transparentes e condutores, ou outras aplicações eletrônicas, é importante que os filmes sejam uniformes, reprodutíveis, apresentem alta transmitância e baixa resistividade elétrica.

Neste trabalho, filmes finos de GO foram produzidos por meio da técnica convencional de spray e em um sistema com spray ultrassônico, com o objetivo de comparar os dois métodos.

\section{MATERIAIS E MÉTODOS}

\subsection{Materiais}

Neste trabalho foram utilizados os seguintes materiais: grafite 99550, da Nacional de Grafite; ácido sulfúrico P.A. 98\% $\left(\mathrm{H}_{2} \mathrm{SO}_{4}\right)$ B Herzog; nitrato de sódio P.A. $\left(\mathrm{NaNO}_{3}\right)$ Vetec; permanganato de potássio $99,3 \%\left(\mathrm{KMnO}_{4}\right)$ Vetec; peróxido de hidrogênio P.A. 32\% $\left(\mathrm{H}_{2} \mathrm{O}_{2}\right)$ Vetec; água deionizada; hidróxido de amônio P.A. 29\% ( $\left.\mathrm{NH}_{4} \mathrm{OH}\right)$ Vetec; ácido ascórbico (AA) P.A. $99 \%\left(\mathrm{C}_{6} \mathrm{H}_{8} \mathrm{O}_{6}\right)$ Synth; PSS (poli (4-estirenosulfonato de sódio)) 30\% Aldrich. 


\subsection{Métodos}

\subsubsection{Produção da dispersão de GO e dos filmes}

O GO foi produzido seguindo o método de Hummers modificado [11], partindo-se de grafite em flocos (99550, da Nacional de Grafite), de acordo com as etapas: intercalação (adição de $\mathrm{NaNO}_{3}$ e $\mathrm{H}_{2} \mathrm{SO}_{4}$ ), oxidação (adição de $\mathrm{KMnO}_{4}$ ) e esfoliação (etapas de lavagem / centrifugação).

Para a fabricação dos filmes pelo método de spray foram empregados dois equipamentos diferentes. Primeiramente utilizou-se um equipamento comercial de spray ultrassônico (Exacta Coat - SONO TEK Corporation) com agulha interna de $100 \mu \mathrm{m}$ e frequência de $120 \mathrm{kHz}$, disponível no Centro de Tecnologia da Informação Renato Archer (CTI Renato Archer), conforme pode ser observado na Figura 1.

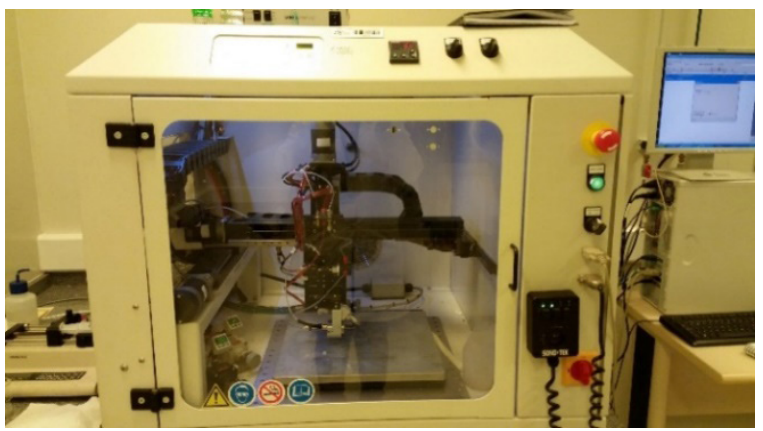

Figura 1: Equipamento industrial para deposição por spray ultrassônico.

Como as folhas de óxido de grafeno apresentavam tamanho da ordem de algumas centenas de $\mu \mathrm{m}$, o que resultava em entupimento do bocal, a dispersão foi sonicada com o auxílio de um ultrassom de ponteira durante duas horas, para diminuição do tamanho das folhas e consequentemente, do número de camadas.

Para a deposição foram utilizados os seguintes parâmetros: concentração da dispersão de $1,0 \mathrm{mg} / \mathrm{mL}$; volume de solução de $30 \mathrm{ml}$; taxa de deposição de $100 \mu \mathrm{m} / \mathrm{min}$; temperatura do substrato de $90^{\circ} \mathrm{C}$; de 5 até 35 camadas.

Os filmes também foram fabricados em um sistema de deposição de spray convencional, montado no Instituto Militar de Engenharia (IME), utilizando-se um aerógrafo Steula BC 66-08, possuindo bico com furo de 0,8 mm; uma placa quente para aquecimento dos substratos; circuito elétrico, para controle do tempo de deposição; um acionador magnético composto por solenoide e pistão, para ativação do gatilho e um programa de computador em linguagem $\mathrm{C}++$. O sistema de deposição automatizado pode ser observado na Figura 2.

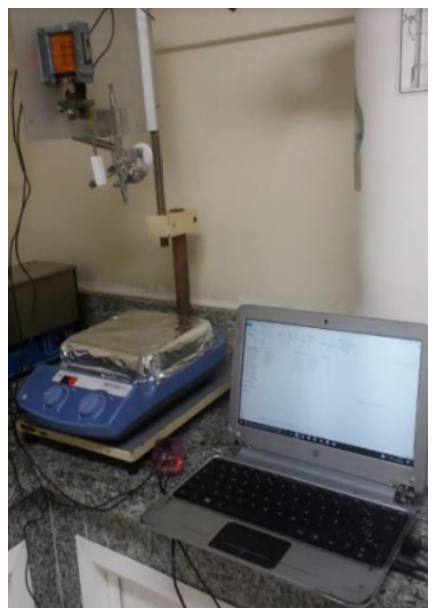

Figura 2: Configuração do sistema de spray convencional automatizado desenvolvido no IME. 
Os parâmetros empregados para as deposições foram: concentração da dispersão de $1 \mathrm{mg} / \mathrm{mL}$; pressão do gás de arraste de 20 psi; distância do substrato até ponteira de $20 \mathrm{~cm}$; temperatura do substrato de $90{ }^{\circ} \mathrm{C}$, tempo de deposição de $0,5 \mathrm{~s}$; tempo de secagem entre camadas de 30 s e 5 até 40 camadas.

Após as deposições, as amostras foram submetidas a recozimento a $650{ }^{\circ} \mathrm{C}$ durante 30 minutos em atmosfera redutora de $\mathrm{H}_{2}$, o que resulta em processo de redução do $\mathrm{GO}$, gerando um filme de rGO.

Os filmes obtidos foram caracterizados com respeito à espessura, resistividade elétrica, transmitância e morfologia, com o intuito de traçar uma análise comparativa entre técnicas empregadas, levando-se em conta a eficiência, simplicidade e viabilidade de cada uma.

\subsubsection{Caracterizações}

A perfilometria de contato foi utilizada para a obtenção da espessura dos filmes produzidos neste trabalho. O equipamento utilizado foi o Dektak 150 da fabricante Veeco, no Laboratório de Filmes Finos (LFF) do IME. Os parâmetros empregados nas análises foram: modo Hills \& Valleys, comprimento de varredura de $1000 \mu \mathrm{m}$, duração de 60 segundos, força $1 \mathrm{mg}$, alcance de $65,5 \mu \mathrm{m}$.

As medidas de resistência de folha foram realizadas pela técnica de quatro pontas posicionadas simetricamente no formado de um quadrado, seguindo o método de Van der Pauw. A caracterização foi realizada no LFF do IME, utilizando o equipamento Hall HL 5500, da fabricante BIO-RAD.

A transmitância foi realizada no LFF do IME, empregando-se o espectrofotômetro CARY 5000, da fabricante VARIAN. As análises foram obtidas para comprimentos de onda de $400 \mathrm{~nm}$ até $800 \mathrm{~nm}$.

Para caracterização morfológica dos filmes, também foi empregado o microscópio QUANTA FEG, marca FEI no IME. Os parâmetros utilizados foram: aumentos de $200 \times, 500 \times, 1000 \times, 2000 \times, 3000 \times$ e $6000 \times$; tensão de 5 kV, spot size 4 e distância de trabalho variável entre 8 e $15 \mathrm{~mm}$. As análises de espectroscopia de raios X por dispersão de energia (EDS) foram realizadas com o auxílio do mesmo equipamento.

\section{RESULTADOS E DISCUSSÃO}

A Figura 3 apresenta as fotografias de amostras obtidas pelos dois processos de spray, sendo que no método ultrassônico foram utilizadas 35 camadas, enquanto no convencional foram 5 camadas. Nota-se que ambas são uniformes e contínuas do ponto de vista macroscópico, sendo que visualmente a diferença entre as duas é irrelevante.

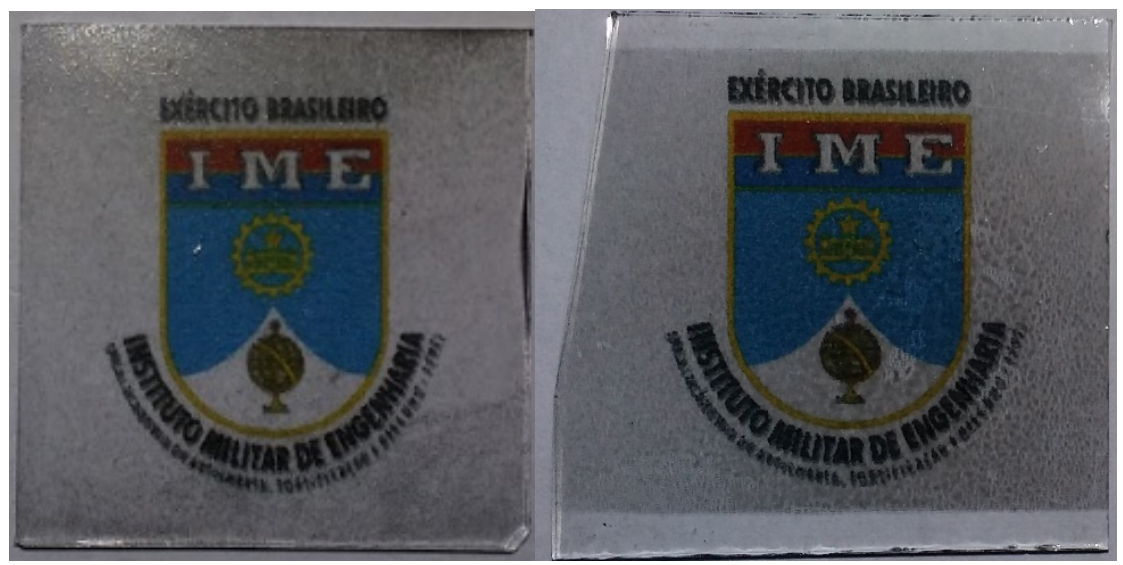

Figura 3: a) amostra produzida por spray ultrassônico; b) amostra produzida por spray convencional.

O primeiro ponto a ser destacado é que, apesar de o equipamento desenvolvido no IME ser uma adaptação muito mais simples e financeiramente mais acessível que o equipamento comercial, os filmes produzidos são visualmente análogos, do ponto de vista macroscópico.

Outro aspecto é a diferença de tempo de produção: enquanto as amostras produzidas por spray ultrassônico levaram 168 min para conclusão, as amostras produzidas por spray convencional foram concluídas em 2,5 min.

Também é interessante destacar que diversos outros parâmetros foram testados para a produção das amostras. Os filmes produzidos no spray ultrassônico com menor número de camadas ( 5 camadas - Figura 4a), apesar de visualmente 
não mostrarem descontinuidade, apresentaram resistência de folha muito elevada, indicando uma descontinuidade elétrica, o que pode ser explicando por uma ausência de contato elétrico entre as folhas do material, conforme medidas de resistividade elétrica realizadas (seção 3.2). Já os filmes produzidos por spray convencional com 30 camadas (Figura 4b), com maior espessura $(784 \pm 36 \mathrm{~nm})$, são inadequados para uso como contato transparente, já que visualmente a transmitância na faixa do visível é próxima a zero.

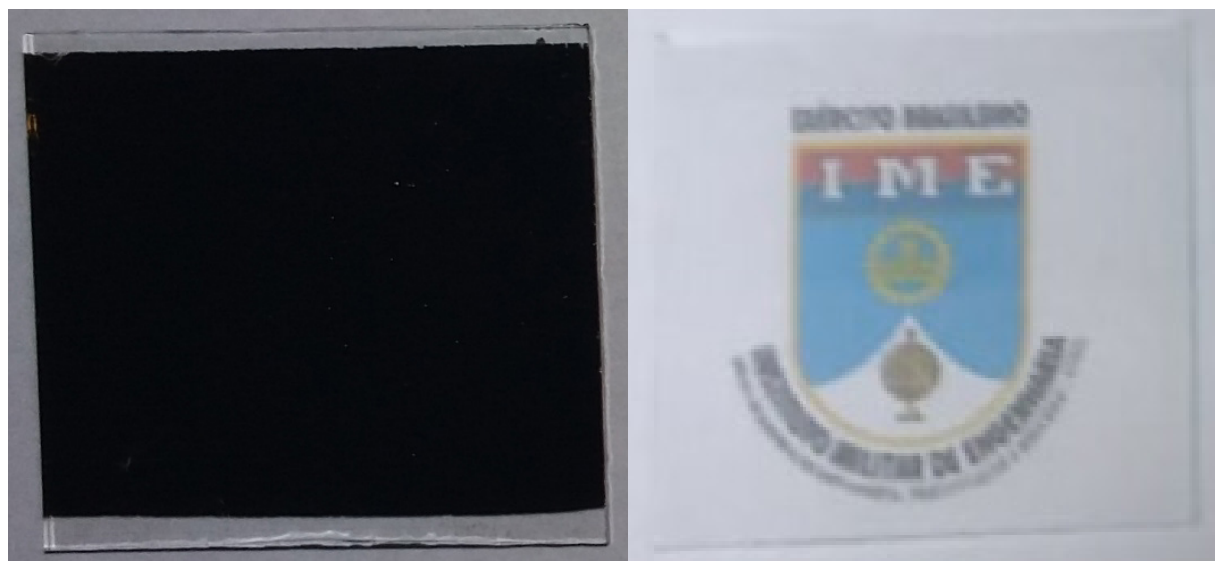

Figura 4: a) filme descontínuo produzido por spray ultrassônico; b) filme espesso produzido com 30 camadas de spray convencional.

\subsection{Espessura}

Os resultados das análises de espessura podem ser observados na Tabela 1. Foram analisadas duas amostras idênticas (elaboradas com os mesmos parâmetros de deposição), produzidas em cada um dos equipamentos. As amostras produzidas spray ultrassônico são identificadas como SU 1 e SU 2 (35 camadas cada), já as amostras produzidas por spray convencional são identificadas como SC 1 e SC 2 (5 camadas cada).

Tabela 1: Espessuras dos filmes estudados.

\begin{tabular}{c|c}
\hline AMOSTRA & ESPESSURA (nm) \\
\hline SU 1 & $921 \pm 22$ \\
\hline SU 2 & $1223 \pm 54$ \\
\hline SC 1 & $87 \pm 2$ \\
\hline SC 2 & $127 \pm 9$ \\
\hline
\end{tabular}

Foram realizadas três medidas em três pontos distintos de cada amostra, com o intuito de verificar a variação da espessura ao longo dos filmes. Observa-se um desvio padrão maior em relação às medidas realizadas nas amostras produzidas por spray ultrassônico, o que provavelmente está relacionado à maior espessura dos filmes ou menor uniformidade da deposição.

\subsection{Resistência de folha}

Os resultados obtidos para as medidas de resistência de folha, de duas amostras produzidas por spray ultrassônico com 35 camadas e duas amostras pelo método convencional com 5 camadas, podem ser observados na Tabela 2. Amostras produzidas com menores números de camadas para ambos os métodos de spray mostram resistências superiores a $1 \mathrm{M} \Omega / \square$, impossibilitando seu uso como filmes para contato elétrico. Assim, são destacadas as amostras que apresentam menores resistência de folha.

É possível observar que os filmes obtidos por spray ultrassônico apresentam menores valores de resistência de folha que os obtidos por spray convencional. Essa diferença pode ser associada à maior espessura dos filmes produzidos pelo método ultrassônico, o que resulta em maior contato das folhas de rGO.

Contudo, um fator relevante neste caso é a discrepância de valores obtidos entre amostras iguais, de 42,70\% para as 
amostras produzidas por spray ultrassônico e 60,26\% para as amostras produzidas por spray convencional. Esses dados indicam que, apesar do equipamento de spray convencional ser muito mais simples e acessível, a reprodutibilidade, em relação a resistência de folha, é relativamente próxima.

Tabela 2: Resistência de folha dos filmes estudados.

\begin{tabular}{|c|c|}
\hline AMOSTRA & Resistência de Folha $(k \Omega / \square)$ \\
\hline SU 1 & 3,04 \\
\hline SU 2 & 7,12 \\
\hline SC 1 & 15,60 \\
\hline $\mathrm{SC} 2$ & 9,40 \\
\hline
\end{tabular}

\subsection{Transmitância}

A Figura 5 apresenta as curvas de transmitância obtidas para as amostras estudadas. É possível notar que as amostras produzidas por spray convencional apresentam maior transmitância, o que está associado à menor espessura das amostras produzidas por esse método.

Em 550 nm, as amostras SU 1 e SU 2 apresentam, respectivamente, 50,87\% e 37,92\% de transmitância; para as amostras SC 1 e SC 2, as transmitâncias a 550 nm são, respectivamente, 61,97\% e 58,73\%. Essas variações de transmitâncias podem ser explicadas com base na dependência exponencial da transmitância em relação à espessura. Segundo a lei de Lambert-Beer, que pode ser observada na Eq. 1, a intensidade da luz transmitida diminui à medida que a espessura do filme aumenta. Nessa equação, é a intensidade inicial da luz incidente, é a intensidade da luz transmitida, é o coeficiente de atenuação (absorção) linear e é a espessura do meio absorvente [12].

$$
\mathrm{I}=\mathrm{I}_{0} \cdot \mathrm{e}^{-\mu .1}
$$

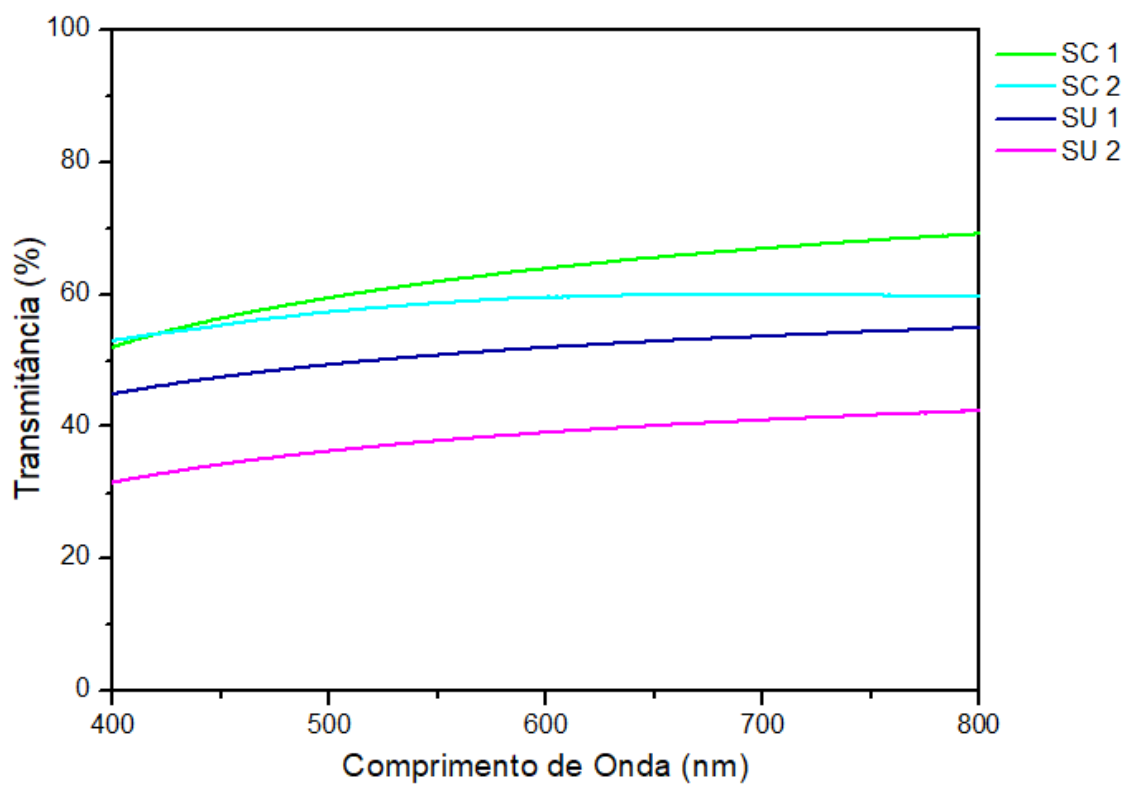

Figura 5: Transmitância dos filmes estudados.

\subsection{Microscopia eletrônica de varredura (MEV)}

A Figura 6 exibe as microscopias obtidas por MEV, respectivamente para a amostra SU 1 (a) e SC 1 (b). Observa-se uma diferença em relação à morfologia dos filmes; enquanto SU 1 apresenta uniformidade, com maior presença de folhas de óxido de grafeno reduzido de dimensões nanométricas, SC 1 tem presença de folhas de rGO de maior dimensão lateral (micrométricas). Isso se deve ao fato de o método de spray ultrassônico ser baseado em transporte de gotículas com tamanhos de poucos micrômetros (em função inversa da frequência do ultrassom), enquanto no spray convencional são arrastadas gotículas com dezenas a centenas de micrômetros, o que resulta em transporte de folhas maiores. 
Os resultados das análises por EDS podem ser observados na Tabela 3. Constata-se que, apesar de produzidas em equipamentos distintos, as amostras apresentam composições similares, sendo a maior concentração de C na amostra SU associada à maior espessura do filme, e os elementos $\mathrm{Na}, \mathrm{Mg}, \mathrm{Al}, \mathrm{Si}$ e $\mathrm{Ca}$, associados ao substrato de vidro, e os elementos como $\mathrm{Cd}, \mathrm{S}$ e Te a possíveis contaminações do forno usado para recozimento das amostras.

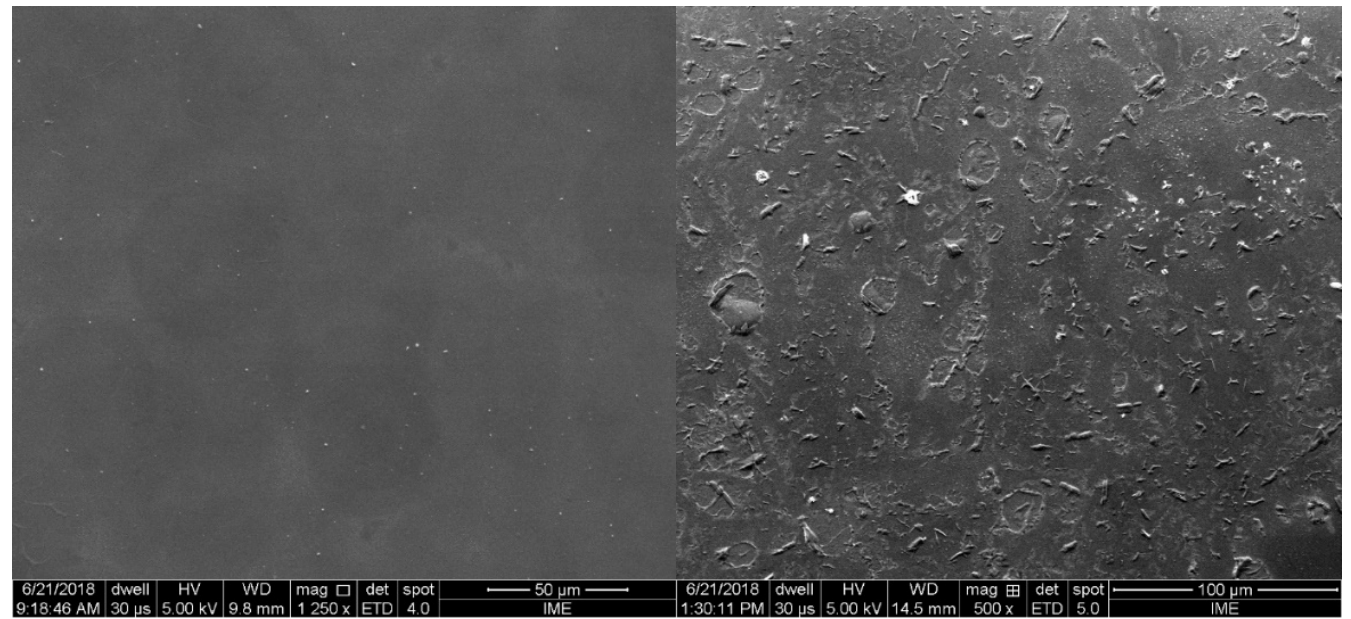

Figura 6: MEV das amostras estudadas: a) SU 1 e b) SC 1.

Tabela 3: Resultados de EDS para as amostras estudadas.

\begin{tabular}{c|c|c}
\hline ELEMENTO & SU 1 (\%) & SC 1 (\%) \\
\hline $\mathrm{C}$ & 8,55 & 4,50 \\
\hline $\mathrm{O}$ & 48,58 & 51,92 \\
\hline $\mathrm{Na}$ & 8,02 & 8,46 \\
\hline $\mathrm{Mg}$ & 1,80 & 1,69 \\
\hline $\mathrm{Al}$ & 0,54 & 0,53 \\
\hline $\mathrm{Si}$ & 26,97 & 27,26 \\
\hline $\mathrm{Ca}$ & 3,15 & 2,56 \\
\hline $\mathrm{Cd}$ & 0,02 & 0,01 \\
\hline $\mathrm{Te}$ & 0,42 & 0,44 \\
\hline $\mathrm{W}$ & 1,96 & 2,27 \\
\hline $\mathrm{S}$ & - & 0,36 \\
\hline
\end{tabular}

\section{CONCLUSÕES}

Por meio das análises realizadas nos filmes preparados nos dois equipamentos, foi possível traçar algumas considerações a respeito da eficiência e produtividade dos métodos utilizados:

1. O equipamento comercial de spray ultrassônico foi capaz de produzir diversas amostras simultaneamente, com precisão e qualidade;

2. O equipamento de spray convencional, apesar de ser um dispositivo muito mais simples e barato, foi capaz de produzir filmes com qualidade similar e, em alguns casos, até mesmo superior aos obtidos por meio do equipamento de spray ultrassônico, resultando em filmes de maior transmitância ( $60 \%)$ mantendo uma resistência de folha não muito alta $(\sim 10 \mathrm{k} \Omega / \square)$;

3. O spray convencional ainda apresenta como vantagens a possibilidade de produzir amostras de GO com tamanho de folha grande $(\mu \mathrm{m})$ em relação ao spray ultrassônico, o que é interessante para aplicações eletrônicas; também devem ser destacados o menor custo, fácil manutenção e menor tempo de produção. 


\section{AGRADECIMENTOS}

Os autores agradecem a Anderson Menezes Lima, pelo auxílio na automatização do sistema de spray; à Nacional de Grafite, pelo fornecimento do grafite para produção do óxido de grafeno; à Coordenação de Aperfeiçoamento de Pessoal de Nível Superior (CAPES) e à Fundação de Amparo à Pesquisa do Estado do Rio de Janeiro (FAPERJ) pelo apoio financeiro.

\section{BIBLIOGRAFIA}

[1] TONG, Y., BOHM, S., SONG, M. "Graphene based materials and their composites as coatings", Austin Journal of Nanomedicine \& Nanotechnology, v. 1, n. 1, pp 1003-1019, Dec. 2013.

[2] MODESTO-LÓPEZ, L., MIETTINEN, M., RIIKONEN, J., et al., "Films of graphene nanomaterials formed by ultrasonic spraying of their stable suspensions”, Aerosol Science and Technology, v. 49, pp 45-56, Dec. 2014.

[3] PU, J., WAN, S., LU, Z., et al., "Controlled water adhesion and electrowetting of conducting hydrophobic graphene/ carbon nanotubes composite films on engineering materials", Journal of Materials Chemistry A, v. 1, pp 1254-1260, Nov. 2012.

[4] LI, P., HUANG, T., WHITE, K., et al., "Spray-coated epoxy barrier films containing high aspect ratio functionalized graphene nanosheets”, RSC Advances, v. 5, n 124, pp 102633-102642, Nov. 2015.

[5] IBRAHIM, A., LIN, S. "Synthesis of graphene oxide membranes on polyester substrate by spray coating for gas separation”, Chemical Engineering Science, v. 190, pp 312-319, Nov. 2018.

[6] MOON, I., KIM, J., LEE, H., et al., "2D Graphene oxide nanosheets as an adhesive over-coating layer for flexible transparent conductive electrodes", Scientific Reports, v. 3, n. 1112, pp 1-7, Jan 2013.

[7] BAYER, T., SELYANCHYN, R., FUJIKAWA, S., et al., "Spray-painted graphene oxide membrane fuel cells", Journal of Membrane Science, v. 541, pp 347-357, Nov. 2017.

[8] NAGAVOLU, C., SUSHIMATHA, K., RAGHAVENDER, M., et al., "Pt-free spray coated reduced graphene oxide counter electrodes for dye sensitized solar cells”, Solar Energy, v. 137, pp. 143-147, Aug. 2016.

[9] SHI, H., WANG, C., SUN, Z., et al., "Transparent conductive reduced graphene oxide thin films produced by spray coating”, Science China Physics, Mechanics \& Astronomy, v. 58, n. 1, pp 1-5, Jan. 2015.

[10] TUNG, T., ALATAIBI, F., NINE, M., et al., "Engineering of highly conductive and ultra-thin nitrogen-doped graphene films by combined methods of microwave irradiation, ultrasonic spraying and thermal annealing", Chemical Engineering Journal, v. 338, pp 764-773, Apr. 2018.

[11] ROURKE, J., PANDEY, P., MOORE, J., et al., "The real graphene oxide revealed - stripping the oxidative debris from the graphene-like sheets”, Angewandte Chemie International Edition, v. 50, n. 14, pp. 3173-3177, Feb. 2011.

[12] MOSOROV, V. "The Lambert-Beer law in time domain form and its application", Applied Radiation and Isotopes, v. 128, pp 1-5, Jun. 2017.

\section{ORCID}

Andreza Menezes Lima Thebano Emílio de Almeida Santos Rodrigo Amaral de Medeiro Leila Rosa de Oliveira Cruz Wagner Anacleto Pinheiro https://orcid.org/0000-0002-5086-6051

https://orcid.org/0000-0001-5473-1376

https://orcid.org/0000-0002-0214-5209

https://orcid.org/0000-0003-2277-0471

https://orcid.org/0000-0003-0560-528X 